\title{
TIEMPO Y ANIQUILACIÓN: CUESTIONES EN TORNO AL LEGADO ARISTOTÉLICO EN LA VISIÓN TOMISTA DE LA TEMPORALIDAD
}

\section{TIME AND ANNIHILATION: ISSUES CONCERNING THE ARISTOTELIAN LEGACY IN THE THOMIST VISION OF TEMPORALITY}

\author{
Ana Maria Carmen Minecan ${ }^{1}$ \\ (Universidad Complutense de Madrid)
}

Recibido: 21/ 4/ 2016

Aceptado: 25/ 6/ 2016

Resumen: El presente artículo analiza el influjo de la doctrina aristotélica en las reflexiones de Tomás de Aquino en torno a la infinitud del tiempo y el movimiento. Se estudian las tres nociones de temporalidad introducidas por el Aquinate - tiempo, eternidad y evo- para salvar los problemas derivados de la filosofía del Estagirita, la aplicación de las premisas de la Física y el Acerca del Cielo en la explicación de la aniquilación de las entidades sublunares y los cuerpos celestes, y el uso de la teoría aristotélica del movimiento en la explicación del Juicio final. Palabras clave: Aristóteles, Tomás de Aquino, tiempo, eternidad, aniquilación.

\begin{abstract}
This article analyzes the influence of the Aristotelian doctrine in the reflections of Thomas Aquinas around the infinity of time and movement. It studies the three notions of temporality introduced by Thomas Aquinas -time, eternity and evo- to save the problems arising from the philosophy of Aristotle, the application of the premises of Physics and On the Heavens in the explanation of the annihilation of the sublunat entitiees and celestial bodies, and the use of Aristotelian theory of motion in the explanation of the last Judgment. Keywords: Aristotle, Thomas Aquinas, time, eternity, annihilation.
\end{abstract}

1. (aminecan@filos.ucm.es) Doctora en Filosofía por la Universidad Complutense de Madrid, actualmente investigador contratado en el ERC-THESIS PROJECT $\mathrm{n}^{\circ} 313339$ "Theology, Education, Scholastic Institution and Scholars-network: dialogues between the University of Paris and the new Universities from Central and Eastern Europe during the Late Middle Ages" perteneciente al Institut de Recherche et d'histoire des textes CNRS Paris. Es autora de numerosos trabajos académicos acera de la asimilación de la física aristotélica y la filosofía de la naturaleza en la Baja Edad Media y la temprana Modernidad publicados en revistas como Anales del Seminario de Historia de la Filosofía, Hybris, Svumma, etc. 


\section{La infinitud del tiempo en el debate antiaristotélico del siglo XIII}

El siglo XIII significó el comienzo de un profundo cambio en el pensamiento filosófico y científico occidental. La recuperación de la práctica totalidad del corpus aristotélico -desconocido para los pensadores tardomedievales a excepción de los tratados de lógica- gracias a la labor de traducción de los textos árabes en Toledo, Salerno y Oxford, dio lugar a una severa crisis en el pensamiento cristiano latino que tuvo que enfrentarse a un conjunto de desafíos que ponían en cuestión las bases mismas de su doctrina.

El análisis de este impacto ha recibido numerosos estudios por parte de la historiografía contemporánea en lo que hace a sus aspectos metafísicos, políticos y éticos. Sin embargo, los estudios en torno a las transformaciones en el ámbito de la física y su relevancia para la creación de una nueva visión de la naturaleza, predecesora de la gran revolución moderna, son limitados o se refieren, constantemente, a las obras de los historiadores de comienzos del siglo XX.

Nuestra intención, en el presente artículo, será la de esclarecer uno de los puntos clave ${ }^{2}$ de los debates antiaristotélicos de la segunda mitad del siglo XIII, a fin de mostrar en qué medida la recepción de la filosofía de Aristóteles provocó la reinterpretación de toda la tradición filosófica anterior ${ }^{3}$.

Las discusiones en torno a los contenidos de los llamados Libros naturales de Aristóteles en la Europa occidental latina tuvieron como primer escenario la Universidad de París. Una breve pero significativa recepción entusiasta de sus textos fue sucedida por un intenso periodo de condenas -desde 1205 hasta 1277- que nos ofrece pruebas de la conmoción que, en el ámbito intelectual, produjo el pensamiento del Estagirita.

El análisis de dos momentos principales del proceso condenatorio permite determinar el peso de las tesis de carácter físico en los contenidos censurados: las condenas de 1270 y $1277 .^{4}$

En el año 1270 se produjo la primera condena formal de contenidos explícitos que no debían ser enseñados en las universidades, ni defendidos

2. En lo que respecta al debate en torno a la asimilación de los tratados físicos de Aristóteles en el siglo XIII pueden señalarse cinco cuestiones fundamentales que centraron la atención de los pensadores y que se encuentran ejemplificadas en las condenas del Syllabus de 1277: eternidad -condenas $\mathrm{n}^{\circ} 4,5,39,45,46,48,52,70,71,80,87,89,90,91,94,99,101,103,107$ y $192-$, finitud $-\mathrm{n}^{\circ} 29,66,96-$ y unicidad del mundo $-\mathrm{n}^{\circ} 34-$, necesitarismo $-\mathrm{n}^{\circ} 21,38,43$, $52,55,58,63,64,67,106,147-$ y existencia del vacío - ${ }^{\circ}{ }^{49-}$. Cfr. Denifle, H. et Châtelain, E.: Chartularium Universitatis Parisiensis, vol.I, Paris: Delalain, 1889. I, p. 486, nº 432. (A partir de aquí $C U P$.)

3. Con ello nos referimos a la cosmología neoplatónica de corte agustiniano compartida por la mayor parte de los filósofos de la Alta Edad Media, cuyas premisas habían constituido la base del pensamiento cristiano en materia física.

4. Hissette, R.: Enquête sur les 219 articles condamnés à Paris le 7 mars 1277. Paris: Vander-

Thémata. Revista de Filosofía №55 (2017) pp.: 177-196. 
por escrito. Entre las trece proposiciones enunciadas en esta condena, es posible distinguir tres referencias claras a los tratados físicos de Aristóteles y a las consecuencias de su asunción que inciden en el problema de la temporalidad que analizaremos a continuación.

La primera tesis de corte físico fue la siguiente: «4.Todo lo que pasa en el mundo inferior está sometido a la acción necesaria de los cuerpos celestes. $\rangle^{5}$

Si bien su contenido parece hacer referencia a las teorías astrológicas que vinculaban el destino y las acciones humanas con las posiciones de los planetas y constelaciones, el lenguaje empleado en su formulación muestra que se trata, sin embargo, de una referencia directa a la física aristotélica.

Una traducción más precisa nos señala que todas las cosas (omnia), que aquí (hic), en la región inferior (in inferioribus) del cosmos, son hechas (aguntur) están sujetas (subsunt) a la necesidad de los cuerpos celestes (necessitati corporum celestium). Es decir, todos los procesos y entidades que existen y se desarrollan en el mundo sublunar dependen de la necesidad impresa por los movimientos eternos de los cuerpos celestes.

Este no es, sino un claro resumen de la mecánica cosmológica de Aristóteles, en la cual el cosmos se concebía como separado en dos regiones ontológicamente distintas pero relacionadas entre sí por una continuidad dinámica. El contacto entre las distintas esferas transmitía mecánicamente el movimiento recibido desde el primer motor hasta las regiones más inferiores del mundo de los compuestos mixtos.

La explicación que podemos ofrecer a la condena de este enunciado es doble. Por un lado, se subraya la incompatibilidad de la libre voluntad divina con la necesidad cosmológica defendida por Aristóteles y, por el otro, se rechaza la vinculación directa entre el mundo sublunar y el mundo de los entes espirituales a fin de mantener vigentes las jerarquías clásicas del neoplatonismo.

La segunda tesis condenada en 1270, fue la siguiente: «5. El mundo es eterno.» ${ }^{6}$. El origen aristotélico de este segundo enunciado es innegable ya que la defensa de la eternidad del mundo constituye uno de los ejes centrales de la física del Estagirita, en la cual se rechaza de forma tajante cualquier tipo de principio respecto al movimiento y al tiempo. Tanto los seres ingenerables e incorruptibles que conforman el mundo supralunar, como el ciclo perpetuo de las transformaciones de los móviles inferiores se hallan sometidos a una temporalidad perpetua, carente de principio y de final. El movimiento circular uniforme de los astros se desliza en una eternidad atemporal, mientras que los movimientos irregulares sublunares

Oyez, 1977, p. 128-132.

5. CUP. I, p. $486, \mathrm{n}^{\circ} 432$.

6. Idem.

Thémata. Revista de Filosofía №55 (2017) pp.: 177-196. 
se repiten incesantemente sustentados por la eternidad de las formas y la materia prima que componen las sustancias hilemórficas. No hubo, para Aristóteles, un momento inicial -ni siquiera en forma de mezcla primigenia o de estado de indeterminación-, y tampoco habrá una descomposición de la armonía natural ya que ningún tipo de causa ${ }^{7}$ puede producir, en su física, una alteración de las leyes regulares que gobiernan el mundo. ${ }^{8}$

Esta estricta visión de la temporalidad cósmica chocaba directamente con las premisas del dogma cristiano que defendían un comienzo temporal del mundo llevado a cabo por la divinidad mediante el acto de creación. ${ }^{9}$

La tercera tesis, coherente con las dos anteriores, que debemos señalar es la siguiente: «6. Nunca ha habido un primer hombre. ${ }^{10}$ Frente a la cosmogonía del Génesis, en el que la realidad natural fue creada en una sucesión temporal en la que es posible distinguir fases distintas respecto a la aparición de los seres en la realidad, el cosmos aristotélico se caracterizaba por una estabilidad férrea. Aristóteles no sólo rechazó un inicio temporal sino cualquier tipo premisa que afirmara que la naturaleza podría haber presentado un estado distinto al actual en un momento del pasado. Todo lo que es y existe, fue y será de la misma manera y en el mismo orden, siempre. Por tanto, no es posible hablar ni de un primer hombre ni tampoco, por extensión, de un primer ejemplar respecto a ninguna de las especies actualizadas en individuos corruptibles.

Si bien las condenas de 1270 dan muestra clara de la importancia de los problemas que la Iglesia comenzó a detectar en la filosofía aristotélica, fue el año 1277 el que simbolizó un cambio radical en la comprensión del aristotelismo.

El Syllabus, decretado por el obispo Esteban Tempier ${ }^{11}$ el 4 de marzo de ese año, contiene 219 condenas en las cuales hay 37 enunciados que se

7. Cfr. Vigo, A.: "Explicación causal y holismo de trasfondo en la filosofía natural de Aristóteles" en Kriterion 51/122, 2010. pp. 587-615.

8. Bechler, Z., Aristotle's theory of actuality, New York: State University of New York Press. pp. 107-110.

9. Seidl, H.: "Is Aristotle's Cosmology and Metaphysics Compatible with the Christian Concept of Creation?", Treschow, M., Otten, W, Hannam, W., (Eds.), Divine Creation in Ancient, Medieval and Early Modern Thought, Leiden-Boston: Brill, 2007. pp.85-100

10. CUP. I, p.486, n 432 .

11. Cfr. Thijssen, J.M.M.H.: "What Really Happened on 7 March 1277? Bishop Tempier's Condemnation and Its Institutional Context" en Sylla, E., McVaugh M., (Eds.), Texts and contexts in Ancient and Medieval Science: Studies on the Occasion of John E. Murdich's Seventieth Birthday, Leiden- New York-Koln: Brill, 1997. pp. 84-114.

Thémata. Revista de Filosofía №55 (2017) pp.: 177-196. 
refieren directamente a distintos aspectos la física de Aristóteles, entre los cuales 19 contienen teorías relacionadas con el problema de la temporalidad.

La tesis que resume con mayor precisión y claridad el pensamiento aristotélico es la siguiente: «87. El mundo es eterno en cuanto a todas las especies contenidas en él; y el tiempo es eterno, así como el movimiento, la materia, el agente y el paciente; y es así porque todo eso procede de la potencia infinita de Dios, y es imposible que haya innovación en el efecto sin una innovación en la causa.» ${ }^{12}$

La primera parte del texto nos informa, punto por punto, de las características principales propias de la naturaleza dibujada por Aristóteles en los tratados de la Física, Acerca del Cielo y Meteorológicos. Como analizaremos más adelante, la eternidad del tiempo y el movimiento constituyen las dos tesis fundamentales de la arquitectura física aristotélica de tal forma que la modificación de alguno de estos puntos implicaría la cancelación del sistema en su conjunto ya que sin ellos no es posible completar la explicación de la mecánica natural desde el punto de vista del Estagirita. ${ }^{13}$

Las siguientes dieciocho tesis complementan, desde distintos puntos de vista, la misma idea, insistiendo en la imposibilidad de aceptar la eternidad del mundo tal y como esta había sido defendida por Aristóteles. ${ }^{14}$

Esta segunda y última fase del proceso condenatorio muestra de forma evidente el tipo de afirmaciones físicas que no podían ser sostenidas

12. CUP, I, p.543, n 473 .

13. Cfr. Dales, R. C.: "Discussions of the Eternity of the World During the First Half of the Twelfth Century"en Speculum, 57, 1982. pp.495-508.

14. «98. El mundo es eterno, porque todo lo que posee una naturaleza por la cual podría ser para siempre en el futuro, tiene una naturaleza por la cual podría haber sido por siempre en el pasado; 99. El mundo, aunque haya sido producido a partir de la nada, no ha sido, sin embargo, producido de nuevo, y aunque haya salido del no-ser para llegar al ser, sin embargo, el no-ser no ha precedido al ser en cuanto a la duración, sino solamente en cuanto a la naturaleza; 4 . Nada es eterno respecto del fin que no sea eterno respecto del principio; 5 . Todas las realidades separadas son coeternas con el primer principio; 45 . El primer principio no es la causa propia de las cosas eternas, si no es metafóricamente, pues él las conserva, esto es, que si él no existiera ellas tampoco existirían; 70. Las inteligencias o sustancias separadas, que se dicen eternas, no tienen una causa eficiente propia, sino metafóricamente, porque tienen una causa que las conserva en el ser, pero no han sido hechas de nuevo, porque entonces serían transmutables; 46 . Así como a partir de la materia no puede hacerse nada sin un agente, así tampoco a partir del agente puede hacerse nada sin una materia; y por esto Dios no es causa eficiente sino de lo que posee el ser en potencia en la materia; 80. Todo lo que tiene materia es eterno, porque lo que no ha sido hecho por transmutación no ha existido anteriormente: por lo tanto, lo que no tiene materia es eterno; 103. La forma que debe devenir y ser en la materia no puede ser producida por aquello que no actúa a partir de la materia; 71. En las sustancias separadas no es posible ninguna transmutación, ni están en potencia respecto a algo, porque son eternas y no tienen materia; 90. El físico debe negar absolutamente la eternidad del mundo porque se apoya sobre causas y razones naturales. En cambio, el creyente puede negar la eternidad del mundo porque se apoya en causas

Thémata. Revista de Filosofía №55 (2017) pp.: 177-196. 
por los pensadores cristianos bajo pena de excomunión. ${ }^{15}$ No obstante, la aceptación de las prohibiciones no fue unánime ni paralizó, a lo largo del siglo XIII, el estudio de los textos aristotélicos.

Un ejemplo fundamental del proceso de asimilación del aristotelismo y de la complejidad de su integración en el pensamiento cristiano fue la obra Tomás de Aquino, en cuyos desarrollos cosmológicos es posible constatar la tensión entre ambos sistemas. Sus textos ofrecen una visión clara de qué elementos de la teoría física aristotélica sobrevivieron y cuáles tuvieron que ser modificados para hacerlos compatibles, aunque de forma polémica, con la cosmovisión medieval.

\section{Tiempo y movimiento en la física de Aristóteles}

Antes de adentrarnos en la exposición de la asimilación tomista de estas cuestiones, resulta conveniente analizar los puntos fundamentales de la física aristotélica que protagonizaron las controversias.

El Estagirita definió la naturaleza como principio del movimiento y del cambio ${ }^{16}$, y parte de la explicación de su defensa de la eternidad de mundo en su conjunto radica precisamente en esta definición. En su camino para lograr las respuestas a las cuestiones fundamentales que pueden

sobrenaturales; 89. Es imposible refutar el argumento del Filósofo en favor de la eternidad del mundo, a menos que digamos que la voluntad del Primero implica imposibles; 91. El argumento del Filósofo demostrando que el movimiento del cielo es eterno no es sofístico; es extraño que hombres profundos no vean esto; 100. Los teólogos que dicen que el cielo está a veces en reposo razonan a partir de una falsa suposición; y decir que el cielo existe, porque no es movido, es afirmar contradictorios; 101. Una infinidad de revoluciones del cielo ha precedido a la revolución actual. No es imposible comprender estas revoluciones infinitas por la primera causa, pero es imposible comprenderlo por un intelecto creado; 107. Los elementos son eternos. Sin embargo, han sido producidos de nuevo en cuanto a la disposición que tienen actualmente; 192. Una forma material no puede ser creada.» CUP, I, p. 543-544, $\mathrm{n}^{\circ} 473$.

15. En la introducción al Syllabus de 1277 se señala de forma clara los castigos que recibirán aquellos que incurran en alguno de los errores condenados: «Nos, por el consejo que nos ha sido dado tanto por los doctores de Teología, como por otros varones prudentes, prohibimos estrictamente que estas u otras cosas semejantes se produzcan y las condenamos totalmente, excomulgando a todos aquellos que han osado profesar, defender o sustentar de algún modo aquellos errores o alguno de ellos, y hacemos lo mismo con los oyentes, a no ser que en el plazo de siete días se presenten ante Nos o ante el canciller de París para revelar esos errores, caso en el que procederemos contra ellos según la gravedad de la culpa a fin de infligir las penas conforme prevé el derecho.» Ídem.

16. «Puesto que la naturaleza es un principio del movimiento y del cambio, y nuestro estudio versa sobre la naturaleza, no podemos dejar de investigar qué es el movimiento; porque si ignorásemos lo que es, necesariamente ignoraríamos también lo que es la naturaleza.» Aristóteles: Física, III 1, $200 \mathrm{~b} 1$.

Thémata. Revista de Filosofía №55 (2017) pp.: 177-196. 
plantearse acerca de los seres naturales, Aristóteles abrió el octavo y último libro de la Física con las siguientes preguntas:

\begin{abstract}
¿Alguna vez fue engendrado el movimiento, no habiendo existido antes, y ha de ser destruido alguna vez, de manera que ya nada estará en movimiento? ¿O no fue engendrado ni será destruido, sino que siempre existió y siempre existirá, y esto inmortal e incesante pertenece a las cosas, como si fuese una vida difundida en todo lo constituido por la naturaleza? ${ }^{17}$
\end{abstract}

Con el objetivo de apuntalar su convicción de la imposibilidad de un origen o un fin temporal para la totalidad del cosmos, Aristóteles caracterizó el movimiento como a "uno de los continuos" acerca de los cuales lo primero que se manifiesta es su infinitud. A esta demarcación del movimiento por medio de la infinitud se unió la definición del mismo como algo indefinido que no puede ser concebido, en sentido absoluto, ni como una potencialidad ni como una actualidad de las cosas. En este sentido, el movimiento, actualidad de lo potencial, es algo esencialmente incompleto y por ello, carente de principio y fin para Aristóteles. ${ }^{18}$

El movimiento, además, no existe fuera de las $\operatorname{cosas}^{19}$, según el Filósofo, pues lo que cambia siempre lo hace sustancial, cuantitativa, cualitativa o localmente, no habiendo nada común a tales cambios que no sea un esto o alguna de las categorías que se predican de la sustancia. Si esto es así, la existencia del movimiento, y de aquello que mueve o es movido, están estrechamente vinculadas. Es decir, si el movimiento es eterno y sin comienzo, deberá haber entes eternos de los cuales se pueda predicar dicho movimiento. El motor inmóvil y todos los astros ingenerables e indestructibles responden a esta exigencia al mantenerse indefinidamente en el ser. Por su parte, en el mundo sublunar si bien no existen entidades que, en sí mismas, permanezcan eternamente sin corromperse, sí puede identificarse como eterno el propio ciclo de generación y destrucción que afecta a los ejemplares de cada especie.

Aristóteles añadió que en todo movimiento hay un moviente primero y algo que es movido, y también un tiempo en el cual, un desde lo que y un hacia lo que. ${ }^{20}$ Entre los distintos tipos de cambios, a su vez, es posible

17. Aristóteles: Física, VIII 1, 250b1-15.

18. Cfr. Cleray, J. J.: Aristotle and Mathematics: Aporetic method in Cosmology and Metaphysics. Leiden: Brill, 1995. pp. 391-392.

19. Aristóteles: Física, III 1, 200b33-35.

20. Aristóteles: Física, V 1, 224b1-3.

Thémata. Revista de Filosofía $\mathrm{N}^{\circ} 55$ (2017) pp.: 177-196. 
distinguir entre el cambio accidental, que se da en todas las cosas y en todos los respectos, y el cambio no accidental.

El cambio no accidental se produce exclusivamente en las cosas contrarias, las intermedias entre las contrarias y en las contradictorias. ${ }^{21}$ Si todo cambio se produce, como ya hemos indicado, desde algo hacia algo ${ }^{22}$, lo que cambia tiene que hacerlo de una de las tres siguientes formas: de sujeto a sujeto (movimiento), de no-sujeto a sujeto (generación), o de sujeto a no-sujeto (destrucción). ${ }^{23}$

Establecidas estas condiciones, Aristóteles mostró las razones por las cuales los movimientos y cambios que contempla su concepción del cosmos han de caracterizarse por la eternidad. En este punto debemos aclarar que, en lo que respecta al mundo supralunar, no cabe hablar de cambio alguno - entendido como movimiento entre contradictorios- sino simplemente de movimientos locales de tipo circular transmitidos mecánicamente hasta la esfera de la Luna.

Los mixtos sensibles sufren dos tipos principales de movimientos -cualitativo y cuantitativo-, así como los cambios de la generación y la destrucción. Sin embargo, el principio de todos los movimientos sublunares se halla, como hemos señalado más arriba, en las translaciones superiores, hecho que convierte al sistema físico aristotélico en una cadena dinámica interconectada en la que el movimiento reverbera desde el primer motor hasta los seres corruptibles. ${ }^{24}$ Este dinamismo manifiesta distintas formas en virtud de la naturaleza de aquello a través de lo cual se propaga. Es decir, a modo de caja de resonancia, la esencia de las cosas produce tonos dinámicos distintos, más simples y perfectos cuanto más sutil es la materia implicada, más irregulares y variados cuanto mayor sea la indeterminación.

Según el Estagirita si cada una de las cosas móviles ha sido generada, entonces con anterioridad a este movimiento tendrá que haber habido otro cambio o movimiento, aquel por el cual fue generado lo que puede ser movido o mover. ${ }^{25}$ Suponer que tales cosas hayan existido siempre, con anterioridad al movimiento, parece algo absurdo porque si entre las cosas móviles y motrices suponemos que en algún tiempo una sea la que primero mueva y otra la que primero es movida, pero en otro tiempo anterior no

21. Íbidem. V I, 224b26-30.

22. Íbidem. V I, 225a1-4.

23. Cfr. Kosman, L.A.: "Aristotle's Definition of Motion” en Phronesis 14 (1), 1969. pp. 40-62.

24. Cfr. Brandie, M., Miller, F. D.: "Teleology and Natural Necessity in Aristotle" en Gerson, Lloyd, P. (Ed.), Aristotle: Logic and metaphysics, New York: Routledge, 1999. p. 69.

25. Cfr. Von Leyden, W., "Time, Number and Eternity in Plato and Aristotle" en Philosophical Quarterly 15 (54) 1964.pp. 35-52.

Thémata. Revista de Filosofía $\mathrm{N}^{\circ} 55$ (2017) pp.: 177-196. 
hay sino reposo, entonces será necesario que haya un cambio anterior al reposo ya que éste no es otra cosa que privación del movimiento. ${ }^{26}$

Por lo que respecta al tiempo, el Estagirita sostuvo que los seres susceptibles de generación y destrucción y, en general, todo lo que es a veces y otras no es, existe necesariamente en el tiempo. Es decir, el ser de cada uno de estos tipos de entidad es medido por el tiempo, hecho del que se sigue que para todo lo que es en el tiempo, cuando existe, tiene que haber necesariamente un tiempo. ${ }^{27}$ Pero si ello es así, según Aristóteles, hemos de admitir un tiempo más grande que el de todo lo que es en el tiempo. ${ }^{28}$ Los límites del tiempo han de ser superiores a aquello que se da actualmente, teniendo que englobar a todo lo que fue pero ya no es y a lo que será pero no es todavía ${ }^{29}$.

El tiempo, según el Estagirita, está esencialmente unido por definición al movimiento de tal forma que no hay tiempo si no hay movimiento. Sin embargo, el tiempo no es movimiento sino algo perteneciente al movimiento. De hecho, Aristóteles señaló que sólo conocemos el tiempo cuando, al determinar el antes y el después, determinamos el movimiento. Es decir, cuando tenemos la percepción del antes y el después -que no son sino atributos de un lugar en virtud de la posición relativa de un cuerpo- del movimiento, decimos que el tiempo ha transcurrido. ${ }^{30} \mathrm{El}$ tiempo queda, de este modo, definido como número ${ }^{31}$-en el sentido de lo numerado- continuo y siempre distinto del movimiento según el antes y el después. ${ }^{32}$

Pero si el movimiento ha sido caracterizado como eterno, el tiempo necesariamente también debe serlo pues, como afirmó el Estagirita, "así como es el movimiento así es también el tiempo". ${ }^{33}$ En este sentido, puesto que el "ahora" es un fin y un comienzo del tiempo, pero no del mismo tiempo, sino fin del que ha pasado y comienzo del que ha de venir, se sigue que el tiempo está siempre en un comienzo y un fin -razón por la cual nos

26. Aristóteles: Física, VIII 1, 251a17-27.

27. Aristóteles: Física, IV 12,221a25.

28. Vigo, A.: "Aristóteles y la infinitud extensiva del tiempo" en Boeri, M.D. (Ed.) Estudios sobre la Física de Aristóteles, Tópicos, México, 30 bis, 2006. pp. 171-205.

29. Cfr. Conen, P.: "Aristotle's Definiton of Time" en The New Scholasticism 26, 1952. pp.441458.

30. Aristóteles: Física, IV 11, 219a22-29.

31. Cfr. Bielke, M. S.: "El tiempo como número y el estrato temporal elemental en Aristóteles" en Thémata. Revista de Filosofía, no 47, (2013), pp. 39-59.

32. Cfr. Bostock, D.: "Aristotle Account of Time", op.cit. Gerson, L. P. Aristotle: Logic and metaphysics. New York: Routledge, 1999. pp.145-155.

33. Aristóteles: Física, IV 13, 222 a31.

Thémata. Revista de Filosofía №55 (2017) pp.: 177-196. 
parece siempre distinto-. Esta es, además, una segunda razón por la cual el tiempo no se extinguirá: porque siempre está comenzando. ${ }^{34}$

A pesar de la fuerza que Aristóteles concede a estas consideraciones, entre todos los pensadores que han hablado acerca del tiempo como algo infinito e ingenerado, reconoció que hubo uno que consideró la posibilidad de hablar de un comienzo: Platón. ${ }^{35}$ En el Timeo, su maestro había hablado de un cosmos generado por el Demiurgo junto con el cual, simultáneamente, era creado el tiempo. ${ }^{36}$

Esta posición resulta, sin embargo, absurda para el Filósofo. Si el tiempo no puede existir ni comprenderse sin el "ahora" y si el "ahora" es un cierto medio o límite entre el tiempo futuro y el tiempo pasado, entonces el tiempo ha de existir siempre. La razón de ello está en el hecho de que si tomamos el extremo del último tiempo, éste ha de ser un "ahora" pero si es un "ahora", entonces ha de haber necesariamente tiempo en ambas direcciones pues este "ahora" es fin del tiempo anterior y principio del que ha de venir. Lo mismo ocurrirá si elegimos el supuesto primer extremo del tiempo. Éste también será un "ahora” y, como tal, implicará la necesaria existencia de un tiempo antes de ese tiempo.

En definitiva, para Aristóteles, no era posible concebir ni un comienzo ni un final para el tiempo ${ }^{37}$ sino que la naturaleza, tomada como un todo, debía ser caracterizada necesariamente bajo la cualidad de lo eterno.

\section{Tiempo, evo y eternidad en Tomás de Aquino}

Tal como hemos podido comprobar en el apartado anterior, la física de Aristóteles defendía de forma inapelable la infinitud del tiempo y el movimiento y, con ello, la eternidad del mundo entendido como escenario del dinamismo incesante de los cuerpos físicos. Estas dos premisas, junto a la conclusión derivada de su asimilación, constituyeron uno de los temas centrales de las reflexiones cosmológica de Tomás de Aquino.

Dejando a un lado la consideración del movimiento analizaremos, a continuación, la concepción tomista del tiempo en la cual es posible comprobar el peso de legado aristotélico, sobre todo en aquellos aspectos en los

34. Íbidem, IV 13,222b1-6.

35. Íbidem. VIII, 1, $215 \mathrm{~b} 15$.

36. Platón: Timeo, 28b-23b.

37. «Además, ¿Por qué el universo se había de destruir precisamente en este punto habiendo existido siempre antes, o se había de generar después de no existir durante un tiempo infinito? En efecto, si no hay mayor motivo ahora que antes y los instantes son infinitos, está claro que existirá durante un tiempo infinito algo generable y corruptible.» Aristóteles: Acerca del cielo, I 12, 283a10-15.

Thémata. Revista de Filosofía ํ55 (2017) pp.: 177-196. 
cuales la aceptación del sistema de Aristóteles llevó a Tomás de Aquino a separarse de los principios de la doctrina cristiana.

La consideración de Dios como creador y origen de toda la realidad llevó a Tomás de Aquino a introducir modificaciones radicales en la concepción del tiempo aristotélico. ${ }^{38}$ Como hemos visto, en el caso del Estagirita el tiempo se caracterizaba por una magnitud tal en la cual debían ser contenidas todas las cosas, incluidas las que existen actualmente, las que han sido y serán. Ello hacía imposible hablar de un comienzo del tiempo ya que cada uno de los "ahoras" que lo constituyen exigían la existencia de un antes y un después en la secuencia.

A pesar de las dificultades que para el cristianismo entrañaba a nivel físico la noción de eternidad, la consideración de esta cualidad como un predicado principal de la divinidad impidió al Aquinate deshacerse de ella. Este problema, unido a las dificultades planteadas por la teoría aristotélica a las nociones de creación y contingencia, le llevaron a concebir tres rangos en lo que respecta al tempus, o mejor dicho, tres medidas de duración relativas cada una a las tres regiones que constituyen su cosmología: Dios, los seres incorruptibles y el mundo de los mixtos naturales.

El primer paso de la argumentación de Tomás de Aquino consistió en refutar la teoría aristotélica por la cual el "ahora" del tiempo permanece invariable mientras dura. Esta permanencia invariable es la definición misma de la eternidad, de tal forma que si el "ahora" del tiempo no es, en esencia, distinto del tiempo mismo, eternidad y tiempo se identificarían ${ }^{39}$. Por otro lado, si la medida del movimiento primero es la medida de todos los movimientos, tal como defendió el Estagirita, la medida del Ser primero ha de ser la misma para todos los entes. De tal forma que si la eternidad se predica de Dios, ésta debería ser también la medida de todos los seres existentes. Ambas cuestiones debían ser refutadas y sustituidas a fin de hacerlas compatibles con la cosmovisión cristiana.

Para llevar a cabo esta tarea, el Aquinate tomó como apoyo la doctrina de Boecio ${ }^{40}$ que le permitió distinguir entre eternidad y tiempo, seña-

38. Nys, D.: "La notion de temps d'après saint Thomas d'Aquin" en Revue néo-scolastique. $4^{\circ}$ año, 13, 1897. pp. 28-43.

39. «Según el Filósofo en el libro IV de la Física, el "ahora" del tiempo permanece invariable mientras éste dura. Pues permanecer invariablemente idéntico durante todo el transcurso del tiempo parece ser o que constituye el concpeto de eternidad. Luego la eternidad es el "ahora" del tiempo. Más como el ahora del tiempo no es en esencia distinto del tiempo mismo, síquese que tiempo y eternidad no son substancialmente distintos.» Tomás de Aquino: Suma teológica, I. Tratado de Dios es uno, traducción comisión presidida por Francisco Barbado Viejo. Madrid: Biblioteca de Autores Cristianos, 2010. 1, q.10 a.4. (A partir de aquí STh)

40. «4. La eternidad es la posesión total y perfecta de una vida interminable. Definición que resultará más clara si la estudiamos en vista a las cosas tempóreas. 5. Todo ser que vive en el tiempo está de continuo yendo desde lo pasado a lo futuro, siendo incapaz de abarcar de una

Thémata. Revista de Filosofía №55 (2017) pp.: 177-196. 
lando que la primera existe de modo simultáneo y toda a la vez, mientras que el segundo se da en un proceso de sucesión. De este modo, el Aquinate diferenció dos formas de duración: a) aquella que constituye la medida del ser inmóvil-Dios y b) aquella que numera el ser de lo que está en movimiento ${ }^{41}$.

(...) si aplicamos la antedicha diferencia, no a la medida, sino a lo medido, tiene el argumento alegado su razón de ser, porque, efectivamente, sólo se mide con el tiempo lo que en el tiempo tiene principio y fin, como dice Aristóteles; y por tanto, en el caso que el movimiento del cielo durase siempre, si bien el tiempo no lo mediría en toda su duración, ya que lo infinito no puede ser medido, sin embargo, mediría cada una de sus rotaciones, pues éstas tienen principio y fin en el tiempo. ${ }^{42}$

Tomás avanzó en la modificación de la posición aristotélica señalando que el "ahora" del tiempo es idéntico, mientras el tiempo dura, sólo desde un punto de vista subjetivo. Es decir, según el Aquinate, esta mera impresión se debe a que el tiempo corresponde al movimiento, el "ahora" del tiempo al móvil y el móvil es el mismo durante todo el transcurso del tiempo. El tiempo es, por tanto para Tomás de Aquino, "el continuo deslizarse del "ahora" en cuanto alterado y numerado por la razón" 43 que mide tanto lo que actualmente cambia como lo que puede cambiar, es decir, el reposo. En cambio, la eternidad permanece la misma tanto en sí como para la razón humana.

Esta distinción entre tiempo y eternidad, sin embargo, no parecía suficiente ya que dejaba sin aclarar qué medida de duración correspondería a las sustancias inmateriales y cuerpos celestes creados a los que, por definición, no se les puede asignar ni la medida propia del Ser absoluto ni tampoco aquella que es propia de los entes móviles. Para solucionar esta dificultad el Aquinate introdujo una tercera medida de duración situada a medio camino entre las dos definidas anteriormente: el evo.

Hay otras [cosas] que están menos alejadas de la permanencia en el ser porque ni su ser consiste en el cambio ni está sujeto a él, aunque tengan aneja a su ser algunas mudanzas, sean éstas actuales o potenciales. Tal sucede a los cuerpos celestes, cuyo

sola vez todda la duración de su existencia. No ha alcanzado aún el día de mañana, cuando ya ha visto perdido el de ayer. En vuestra vida actual sólo vivís el momento presente, rápido y fugaz. 6. Así, un ser sujeto a la ley del tiempo, puede no haber tenido principio y no tener fin, como Aristóteles afirma del mundo; pero no por eso reúne las condiciones necesarias para que se le pueda llamar eterno.» Boecio: La consolación de la filosofía, traducción Masa, P., prólogo y notas, Castaño Piñán, A.. Buenos Aires: Aguilar, 1955.. L.V, prosa 6, 4-6.

41. STh. 1, q.10, a.4.

42. Ídem.

43. STh. 1, q.10, a.4.

Thémata. Revista de Filosofía №55 (2017) pp.: 177-196. 
ser sustancial es inmutable, pero que junto con la inmutabilidad en el ser tienen el poder de cambiar de lugar. (...) Estos son, por tanto, los seres que se miden por el evo, que ocupa una posición media entre el tiempo y la eternidad; pues el ser que la eternidad mide, ni es mudable ni tiene aneja mudanza. ${ }^{44}$

La diferencia que existe entre el evo y la eternidad radica fundamentalmente en que el primero admite el antes y el después, es decir, en él se puede señalar una sucesión diacrónica susceptible de medir cosas sujetas a cambios, mientras que en la segunda estas distinciones son imposibles debido a su naturaleza absolutamente sincrónica. Ambas medidas, en cambio, coinciden en el hecho de que su duración es infinita, frente al tiempo diacrónico limitado tanto en su comienzo como en su final.

Esta caracterización mixta del evo como medida secuencial e infinita permite observar cómo el tiempo aristotélico no desapareció en la obra de Tomás de Aquino sino que se recuperó, bajo un nuevo nombre, como solución a las condiciones establecidas por las Escrituras y la tradición cristiana: una existencia simultánea y absoluta para la divinidad, una duración finita para los seres creados y una medida intermedia dotada de las propiedades del tiempo descrito en la Física para las criaturas espirituales.

\section{La aniquilación y el retorno a la nada}

Si bien estas modificaciones parecían solventar las primeras dificultades derivadas de la física aristotélica, precisamente la referencia al estatus temporal de lo creado -tanto físico como inmaterial- llevó a Tomás de Aquino a abordar dos de las cuestiones introducidas por el cristianismo más complejas de conciliar con la filosofía de Aristóteles: la aniquilación de las criaturas por parte de Dios y el Juicio final defendido en la escatología. ${ }^{45}$

El problema del fin de los tiempos constituía un reto tan difícil como el de la creación ${ }^{46}$ e incluso aún más acuciante, en la medida en que

44. Íbidem. 1, q.10, a.5.

45. Cfr. Weinandy, T., Keating, D., Yocum J.: Aquinas on Doctrine: A cricial introduction, New York: T\&T Clark Ltd, 2004. pp.235-237.

46. Cfr. Hankey, W. J.: "Ab uno Simplicio non est nisi unum: The Place of Natural and Necessary Emanation in Aquinas's Doctrine of Creation" en Treschow, M., Otten, W, Hannam, W., (Eds): Divine Creation in Ancient, Medieval and Early Modern Thought, Leiden-Boston: Brill, 2007 pp. 309-334.

Thémata. Revista de Filosofía №55 (2017) pp.: 177-196. 
el Doctor Angélico aceptó, sin restricciones, la teoría aristotélica según la cual los cuerpos celestes son incorruptibles e indestructibles. ${ }^{47}$

Es decir, si bien, en virtud de la doctrina del Génesis, el Aquinate aceptó un comienzo temporal para la existencia de este tipo de entidades, sus reflexiones en torno al destino final de las mismas se separaron de las posiciones cristianas al tomar por verdaderas las bases de la física aristotélica establecidas al respecto en el Acerca del cielo.

El dogma establecía que Dios, al igual que había tenido la suficiente potencia como para crear el mundo, debía ser capaz igualmente de reducirlo a la nada. No obstante, Tomás de Aquino decidió evitar la atribución de una acción negativa a la divinidad mediante la apelación a un conjunto de tesis que le permitieron mantener tanto la integridad de la bondad divina como la de las partes centrales del sistema aristotélico que estaba aceptando. ${ }^{48}$

El primer paso de su argumentación consistió en la asunción de la tesis aristotélica según la cual los compuestos poseen en sí mismos una tendencia hacia el no-ser y la disgregación de sus partes. Sin embargo, el Aquinate introdujo una modificación fundamental en su planteamiento: mientras que para Aristóteles sólo existía una destrucción parcial, para el dominico hay en las criaturas una inclinación al no-ser absoluto que proviene de su propio origen ontológico último. De forma paradójica, pero consecuente con la tesis agustiniana de que la divinidad creó sin poner nada de sí misma en el proceso, Tomás de Aquino, señaló la nada como algo capaz de dejar una cierta marca en las cosas creadas. Así, el dominico afirmó que dado que las cosas proceden de la nada, hay en ellas una tendencia a regresar a la misma que sólo se ve coartada por la constante acción divina.

Esta interpretación le permitió establecer que si Dios deseara aniquilar la creación, ello no le obligaría a llevar a cabo un acto específico de destrucción -y por ello susceptible de ser calificado de negativo- sino

\section{Aristóteles, Acerca del cielo, I, 10-12.}

48. La interpretación de Tomás de Aquino dista mucho de la acción destructiva que se presenta en el Apocalipsis, texto en el cual se narra detalladamente la aniquilación secuencial y activa de las distintas regiones del cosmos: «El primero tocó la trompeta y cayó sobre la tierra granizo y fuego mezclado con sangre; la tercera parte de la tierra quedó abrasada, la tercera parte de los árboles quedó abrasada y la tercera parte de la hierba verde quedó abrasada. El segundo ángel tocó la trompeta, y una enorme mole de brasas, como una montaña fue lanzada al mar; la tercera parte del mar se convirtió en sangre, pereció la tercera parte de los seres del mar y la tercera parte de las naves fueron destruidas. El tercer ángel tocó la trompeta, cayó del cielo una gran estrella, ardiente como una llama; cayó sobre la tercera parte de los ríos y sobre las fuentes de las aguas. (...) El cuarto ángel tocó la trompeta, y la tercera parte del sol, la tercera parte de la luna y la tercera parte de las estrellas quedaron heridas de muerte de tal manera que se oscureció la tercera parte de las mismas y el día perdió una tercera parte de su esplendor, lo mismo que la noche.» Apocalipsis 8, 7-13.

Thémata. Revista de Filosofía $\mathrm{N}^{\circ} 55$ (2017) pp.: 177-196. 
que simplemente podría limitarse a detener su acción conservadora o, tal como lo expresa el Aquinate, a «dejar de darles el ser». ${ }^{49}$ De esta forma, serían las criaturas por sí mismas, y no por un acto divino, las que acabarían desapareciendo.

El no-ser no tiene directamente causa, porque nada puede ser causa sino en cuanto es ser, y el ser, de suyo es causa de ser. En este sentido, Dios no puede ser causa de la tendencia al no-ser. Mas esta tendencia al no-ser la lleva consigo la criatura misma en cuanto que proviene de la nada. Puede Dios, sin embargo, ser causa indirecta de que las cosas vuelvan a la nada, simplemente con retirar de ellas su acción. ${ }^{50}$

Esta explicación, que podía ofrecer una respuesta al retorno de la nada de los compuestos hilemórficos sublunares, se mostraba absolutamente insuficiente en el caso de los astros. La aceptación por parte del Aquinate de la teoría aristotélica relativa a su incorruptibilidad ${ }^{51}$ le obligaba a admitir que, para dejar de existir, o bien debían ser aniquilados activamente por Dios o, por su propia esencia, jamás dejarían de existir ${ }^{52}$.

En este punto, el Aquinate abrió una dicotomía en su discurso mediante la cual intentó salvar tanto la física aristotélica como las premisas

49. En el artículo tercero de la quinta cuestión del De potentia Dei, Tomás de Aquino precisa esta cuestión señalando que la tendencia a la nada de las criaturas no las acabaría aniquilando mientras la divinidad las conserve en el ser. Es decir, siempre que su existencia siga garantizada por Dios, la propia estructura de la naturaleza se perpetuará sobreponiéndose a dicha inclinación. En este sentido, no son causas físicas las que pueden conducir a la aniquilación de lo natural, sino de orden metafísico relativas a la absoluta dependencia de lo creado -en lo que respecta a su existencia- de la divinidad. "A lo primero hay que decir que si Dios redujera las criaturas a la nada, no sería a causa de la tendencia al no ser; esto no sucedería porque Él mismo causara el no ser de las cosas, sino porque dejaría de darles ser.» Tomás de Aquino: De potentia Dei, 5 La conservación, traducción y notas de Prieto, N., Pamplona: Servicio de Publicaciones de la Universidad de Navarra, 2005. q.5, a.3. (A partir de aquí QDP 5)

50. STh. I, q.104. a.3.

51. «Por tanto, sólo tienen posibilidad de no ser las cosas en las que hay materia sujeta a contrariedad. A las demás cosas, en cambio, según su naturaleza les corresponde la necesidad de ser, excluida de su naturaleza la posibilidad de no ser.» QDP 5, a.3.

52. Si bien, tal como hemos señalado más arriba, existe en las cosas creadas una inclinación al no-ser, dicha tendencia encuentra un contraejemplo en las cosas que han sido creadas con una esencia eterna. Es decir, si bien éstas también dependen en su modo de existencia necesario del apoyo divino - «(...) esto no quita que en ellas la necesidad de ser sea por Dios, porque uno puede ser la causa de la necesidad de otros, como se dice en Metafísica V»-, su herencia ontológica de la nada debe entenderse como cancelada o, de lo contrario, se tendría que afirmar que Dios ha creado esencias eternas en vano. «(...) Dios instituyó cada naturaleza de tal modo, que no le quitó lo que le es propio; por eso se dice en la Glosa a los Romanos XI, 24 que Dios, que es el fundador de las naturalezas, no obra contra las naturalezas, aunque alguna vez en apoyo de la fe obre en las cosas creadas algo por encima de la naturaleza. Pero la eternidad es una propiedad natural de las cosas inmateriales, que carecen de contrarios;

Thémata. Revista de Filosofía №55 (2017) pp.: 177-196. 
de la fe cristiana, señalando que Dios opera sobre los seres creados de dos maneras: secundum naturale cursum o secundum miracolum.

Tomada la cuestión desde el punto de vista del orden natural, el destino de los seres, puede deducirse, según el Aquinate, de su propia naturaleza. De ello se sigue que si la condición natural de una cosa manifiesta su incorruptibilidad, esta cosa no podrá dejar de existir mediante ningún proceso físico.

Para que este tipo de entidades sean aniquiladas, la divinidad ha de romper el orden natural que ella misma ha impuesto al cosmos y llevar a cabo un acto milagroso cuya explicación, de darse, sería completamente inalcanzable para el entendimiento humano. ${ }^{53}$

Es decir, para devolver los cuerpos celestes a la nada Dios debería modificar su propia creación - hecho que entra en colisión con su carácter de creador inteligente- para alterar la naturaleza de aquello que él mismo ha creado en un principio como incorruptible. Ante esta dificultad, el Aquinate decidió decantarse por la solución más lógica y considerar, para no caer en contradicción y en contra de lo establecido en las Escrituras ${ }^{54}$, que ninguna de las cosas creadas será reducida a la nada por Dios.

Tampoco contribuiría a la manifestación de la gracia el que alguna cosa fuese reducida a la nada; por el contrario, el poder y la bondad de Dios se manifiestan más claramente en el hecho de conservar las cosas en el ser. Se debe, pues categóricamente afirmar que nada absolutamente se aniquilará. ${ }^{55}$

porque en ellas no hay potencia para no ser, como se mostró más arriba. Por consiguiente, como al fuego no se le quita la natural inclinación, por la que tiende a subir, así no se les quita a las cosas citadas la eternidad, para reducirlas a la nada.» Ídem, a.4.

53. Cfr. Luck, M.: "Aquinas's miracles and the luciferous defence: The problem of the evil/ miracle ratio" en Sophia 48, 2009. pp. 167-177.

54. En el relato del último juicio del Apocalipsis se distingue, de forma metafórica, entre dos destinos para los seres humanos: la salvación - con la consecuente entrada en la Jerusalén celeste- y la segunda muerte o muerte definitiva que puede comprenderse como un retorno a la nada ejemplificada por el estanque de fuego. «Vi un gran trono blanco y al que estaba sentado sobre él. El cielo y la tierra huyeron de su presencia, sin que se encontrase su lugar. Vi los muertos, grandes y pequeños, en pie delante del trono; entonces fueron abiertos los libros; fue abierto también otro libro, el libro de la vida. Y los muertos fueron juzgados según el contenido de los libros, cada uno según sus obras. El mar devolvió los muertos que guardaba; la muerte y el abismo devolvieron los muertos que guardaban, y cada uno fue juzgado según sus obras. La muerte y el abismo fueron arrojados al estanque de fuego: el estanque de fuego es la segunda muerte. Y el que no fue encontrado escrito en el libro de la vida fue arrojado al estanque de fuego.» Apocalipsis, 20, 11-15.

55. STh. 1, q.104, a.4.

Thémata. Revista de Filosofía $\mathrm{N}^{\circ} 55$ (2017) pp.: 177-196. 


\section{El juicio final y el cese del movimiento}

A pesar de esta afirmación, que rechaza la aniquilación o el retorno a la nada, Tomás de Aquino tuvo que enfrentarse, de modo inevitable, al problema del final del universo creado que se verificaría, según la fe, en el proceso del Juicio final. El núcleo de esta creencia establecía un momento de cierre en el cual la naturaleza física vería extinguidos todos sus procesos para dar paso a una etapa desconocida ${ }^{56}$ respecto al estatus arquitectónico del mundo.

La explicación de las modificaciones de tipo físico que debían tener lugar en el Juicio final fue llevada a cabo por el Aquinate con ayuda de la teoría aristotélica del movimiento. El dominico subrayó, siguiendo las premisas de la física del Estagirita, que en los cuerpos inferiores la generación y la corrupción son causadas por el movimiento del cielo. Por tanto, para que se produzca el cese de los fenómenos que alberga el mundo sublunar, el Juicio final ha de consistir en un proceso mediante el cual se detenga necesariamente su movimiento. Este cese de los ciclos de los astros implicaría la detención de la dinámica del cosmos, hecho que lleva a hablar, de forma coherente con las exigencias aristotélicas, del final del tiempo.

A este hecho, Tomás de Aquino añadió la sorprendente afirmación de la permanencia eterna de la sustancia de toda la creación. Es decir, una vez cesado el movimiento de los cielos, de los elementos y de los procesos de generación y destrucción, permanecerá, no obstante, la esencia de las cosas que han sido hechas como sempiternas: los cuerpos celestes, las formas, los elementos, la materia y las almas de los seres humanos.

\footnotetext{
Ahora bien, los cuerpos celestes tienen naturaleza para ser perpetuos total y parcialmente; sin embargo, los elementos sólo totalmente, porque parcialmente son corruptibles y los hombres, parcialmente, pero no totalmente porque el alma racional es incorruptible, pero el compuesto es corruptible. Por lo tanto, permanecerán en cuanto a la sustancia en aquél último estado del mundo las cosas que de algún modo tienen aptitud para la perpetuidad, supliendo Dios con su poder lo que les falta por su propia debilidad..$^{57}$
}

En cambio, los compuestos - como los animales y las plantas- no permanecerán tras el Juicio final ya que su naturaleza mixta precisa ne-

56. Las Escrituras no contienen textos claros acerca de la configuración del mundo posterior al Juicio final, no obstante, se insiste en diversas ocasiones en la idea de que habrá una nueva tierra tras la destrucción completa de la anterior. "Vi un cielo nuevo y una tierra nueva, porque el primer celo y la primera tierra habían desaparecido; y el mar ya no existía.» Apocalipsis 21, 1-2.

57. Tomás de Aquino: Suma contra los gentiles, I. Libros $1^{\circ}$ y $2^{\circ}$, Dios, su existencia, su

Thémata. Revista de Filosofía $\mathrm{N}^{\circ} 55$ (2017) pp.: 177-196. 
cesariamente de los distintos tipos de movimiento para existir. Por tanto, lo que se producirá será una pérdida absoluta de los accidentes quedando, solamente, la sustancia. Es decir, cesará aquello que el Aquinate denominó "apariencia del mundo".

En este sentido, en términos físicos, el dominico no propuso literalmente un fin de toda la realidad sino más bien un momento de transformación por el cual desaparecerán todas las criaturas de temporalidad limitada, dejando paso a una naturaleza caracterizada esencialmente por la infinitud existencial. Todo lo corruptible, todo lo alterable, todo lo que está destinado a perecer bajo la acción del tiempo desaparecerá manteniéndose, no obstante, el esqueleto básico de la realidad ontológica aristotélica caracterizada como inalterable.

\section{Conclusión}

Tomás de Aquino diferencia, tres tipos de temporalidad -tiempo diacrónico, eternidad y evo- entre los cuales el tercero, empleado para designar la medida cronológica de los cuerpos celestes y las sustancias inmateriales creadas, mantiene las características del tiempo aristotélico: infinitud y distinción entre el antes y el después. Asimismo, frente a las doctrinas contenidas en las Escrituras y a favor de la teoría aristotélica del carácter indestructible de los entres supralunares, defiende la eternidad a parte post de los cuerpos celestes, las formas, la materia y las sustancias espirituales.

El mundo, tal y como Tomás de Aquino defendió también en su opúsculo De aeternitate mundi contra murmurantes, si bien fue creado, es eterno ya que mantiene una forma de existencia a pesar de perder el elemento central que vertebraba el mundo físico aristotélico: el movimiento.

Su anulación definitiva generará un estado de reposo libre de todo rasgo de corporeidad en el cual se producirá un salto hacia lo inmaterial en el que permanecerán, de forma inactiva, los elementos estructurales de la cosmología aristotélica en un estado estacionario, atemporal y eterno.

naturaleza; La creación y las criaturas, introducción Eudaldo Forment, Madrid: Biblioteca de Autores Cristianos, 2007.IV, 97.

Thémata. Revista de Filosofía $\mathrm{N}^{\circ} 55$ (2017) pp.: 177-196. 


\section{Referencias bibliográficas}

Aristóteles: Física, Madrid: Gredos, 2008.

Aristóteles: Acerca del cielo, Meteorológicos, Madrid: Gredos, 2008.

Bechler, Z.: Aristotle's theory of actuality, New York: State University of New York Press, 1995.

Bielke, M. S.: "El tiempo como número y el estrato temporal elemental en Aristóteles" en Thémata. Revista de Filosofía, nº 47, 2013, pp. 39-59.

Boecio: La consolación de la filosofía, traducción Masa, P., prólogo y notas, Castaño Piñán, A.. Buenos Aires: Aguilar, 1955.

Bostock, D.: "Aristotle Account of Time" en Gerson, L. P.: Aristotle: Logic and metaphysics. New York: Routledge, 1999. pp. 148-169

Brandie, M. y Miller, F.D.: "Teleology and Natural Necessity in Aristotle" en Ídem. pp. 75-89.

Cleray, J. J.: Aristotle and Mathematics: Aporetic method in Cosmology and Metaphysics, Leiden: Brill, 1995.

Conen, P. "Aristotle's Definiton of Time" en The New Scholasticism 26, 1952. pp.441-458

Denifle, H. et Châtelain, E.: Chartularium Universitatis Parisiensis, vol. I, Paris: Delalain, 1889.

Dales, R. C.: "Discussions of the Eternity of the World During the First Half of the Twelfth Century" en Speculum, 57, 1982. pp.495-508.

Hissette, R.: Enquête sur les 219 articles condamnés à Paris le 7 mars 1277. Paris: Vander-Oyez, 1977.

Kosman, L.A.: "Aristotle's Definition of Motion" en Phronesis 14 (1), 1969. pp. 40-62.

Luck, M.: "Aquinas's miracles and the luciferous defence: The problem of the evil/miracle ratio" en Sophia 48, 2009. pp. 167-177.

Nys, D.: "La notion de temps d'après saint Thomas d'Aquin" en Revue néo-scolastique. $4^{\circ}$ año, n.13, 1897. pp. 28-43.

Platón: Diálogos IV: Filebo,Timeo, Critias, Cartas, Barcolena: Gredos, 2007.

Seidl, H.: "Is Aristotle's Cosmology and Metaphysics Compatible with the Christian Concept of Creation?" en Treschow, M., Otten, W, Hannam, W., (Eds): Divine Creation in Ancient, Medieval and Early Modern Thought, Leiden-Boston: Brill, 2007. pp.85-100

Thijssen, J.M.M.H., "What Really Happened on 7 March 1277? Bishop Tempier's Condemnation and Its Institutional Context" en Sylla, E., McVaugh M., (Eds.): Texts and contexts in Ancient and Medieval Science: Studies on the Occasion of John E. Murdich's Seventieth Birthday, Leiden- New York-Koln: Brill, 1997. pp. 84-114. 
Tomás de Aquino: Suma teológica, I. Tratado de Dios es uno, traducción comisión presidida por Francisco Barbado Viejo, Madrid: Biblioteca de Autores Cristianos, 2010.

: Suma contra los gentiles, I. Libros $1^{o}$ y $2^{\circ}$, Dios, su existencia, su naturaleza; La creación y las criaturas, introducción Eudaldo Forment, Madrid: Biblioteca de Autores Cristianos, 2007.

: De potentia Dei, 5 La conservación, traducción y notas de Prieto, N., Pamplona: Servicio de Publicaciones de la Universidad de Navarra, 2005

Vigo, A.: "Explicación causal y holismo de trasfondo en la filosofía natural de Aristóteles" en Kriterion 51/122 , 2010. pp. 587-615.

-: "Aristóteles y la infinitud extensiva del tiempo" en Boeri, M.D. (Ed.) Estudios sobre la Física de Aristóteles, Tópicos, México, 30 bis, 2006. pp. 171-205.

Von Leyden, W.: "Time, Number and Eternity in Plato and Aristotle" en Philosophical Quarterly 15 (54) 1964.pp. 35-52.

Weinandy, T., Keating, D., Yocum J.: Aquinas on Doctrine: A cricial introduction. New York: T\&T Clark Ltd, 2004. 